\title{
Quasiparticle Random Phase Approximation with inclusion of the Pauli Exclusion Principle
}

\author{
F. Šimkovic ${ }^{1 *}$, A. A. Raduta ${ }^{1 \dagger}$, M. Veselský2 ${ }^{\ddagger}$, and Amand Faessler ${ }^{1 \S}$, \\ 1. Institute of Theoretical Physics, University of Tuebingen, D-720 76 Tuebingen, Germany \\ 2. Cyclotron Institute, Texas A\&M University, College Station, TX-77843, USA
}

(September 13, 2018)

\begin{abstract}
Limitations of the Quasiparticle Random Phase Approximation (QRPA) are studied within an exactly solvable model, with a two body interaction of Fermi type. A special attention is paid to the violation of the Pauli exclusion principle (PEP) in solving the QRPA equation. A comparison of the exact solution, obtained by the diagonalization of a schematic nuclear Hamiltonian and those obtained within the standard QRPA, the renormalized QRPA, the QRPA with pertubative treatment of the PEP and the QRPA with exact consideration of the PEP, is presented. The agreement quality is judged in
\end{abstract}

\footnotetext{
* On leave from: Department of Nuclear physics, Comenius University, SK-842 15 Bratislava, Slovakia, e-mail: simkovic@fmph.uniba.sk

${ }^{\dagger}$ On leave from: Institute of Physics and Nuclear Engineering, Bucharest, POB MG6, Romania and Dept. of Theoretical Physics and Mathematics, Faculty of Physics, Bucharest University, POB MG 11, Romania, e-mail: raduta@theor1.theory.nipne.ro

$\ddagger$ On leave from: Institute of Physics of Slovak Academy of Sciences, SK-842 28 Bratislava, Slovakia, e-mail: fyzimarv@savba.sk

§e-mail: amand.faessler@uni-tuebingen.de
} 
terms of the quasiparticle number operator matrix elements in the ground state and in the first excited states, of the $\beta$ transition amplitudes, of the Ikeda sum rule and of the nuclear matrix element for the double beta decay. We have found that restoring the PEP, the QRPA solutions are considerably stabilized and a better agreement with the exact solution is obtained.

Typeset using REVTEX 


\section{INTRODUCTION}

The Quasiparticle Random Phase Approximation (QRPA) has been found to be a powerful method for describing many-body systems. Due to its simplicity, the proton-neutron QRPA is the nuclear structure method which has been most frequently used to interpret some nuclear structure aspects of the beta $(\beta)$ and double beta $(\beta \beta)$ decay for open shell systems [1-10]. The QRPA provides a description of excited states by including some nucleon-nucleon correlations in the ground state.

The QRPA equations are derived directly from the equation of motion. In deriving the QRPA equations two basic approximations are adopted: (i) The operator, which determines the excited state, is taken as linear superposition of two creation and two annihilation quasiparticle operators by considering the BCS basis as reference. (ii) The commutator of bifermion operators is replaced by its expectation value in the BCS ground state. This is usually called the "quasiboson approximation" (QBA). The QBA violates the Pauli exclusion principle (PEP) and this affects severely the theory. The terms which are left out by the QBA become more and more important when the ground state correlations are increased which results in a collapse of the QRPA solution. The approach based on the two approximations mentioned above will be conventionally called "the standard QRPA approach".

Recently, the instability of the QRPA solution, caused by the PEP violation received much attention from the experts in the field. In order to improve the reliability of the standard QRPA description of the nuclear transitions, the renormalized version of the QRPA (RQRPA), which take into account the PEP in an approximate way, has been formulated 11.,12 and applied to the $\beta$ and $\beta \beta$ decay problems [13 15. Indeed, the RQRPA does not collapse within the physical range of the interaction strength parameters. However, avoiding the collapse in the RQRPA a price had to be paid, namely the violation of the Ikeda sum rule [16, 177.

There is a constant interest in studying the physical consequences of violating the PEP by the QRPA solutions. Some definite conclusions can be drawn by using solvable models, 
like, for example, the extensions [17,18] to proton-neutron systems of Lipkin or Moszkowski models [19,20], as they simulate the realistic cases either by analytical solutions or by a minimal computational effort. It is worthwhile mentioning that the study of different manybody approximations within schematic models was always of great interest and moreover it is currently considered of a major importance [17,21, 29, 31].

The improvement of the PEP obedience, within the QRPA, can be achieved in two ways: (i) By a mapping technique the whole theory can be formulated in a boson picture. Such an approach has been outlined for the proton-neutron monopole Lipkin model in Ref. [22 26]. (ii) One can remain within the fermionic space and derive the elements of the QRPA equation, at least, perturbatively. Usually approximations violating the PEP are tested for Fermi transition since for this case the schematic models are simple and solvable [17,22 26,31]. Recently a more general solvable model appeared [27,28] that exploits the $\mathrm{SO}(8)$ symmetry to include simultaneously the Fermi and Gamow-Teller transitions. Such a model is devoted to an extensive treatment of the proton-neutron pairing interaction.

The goal of this work is to discuss some limitations of the standard QRPA approach, concerning the PEP violation. We shall follow the second possibility mentioned above and introduce new extensions of the standard QRPA approach within the proton-neutron monopole Lipkin model and point out some implications for realistic calculations. The newly introduced approximations will be compared with the exact results, revealing, in this way, the limits of the approximations. Our work completes the discussion of the Refs. [17,22, 26] in which the same schematic model was considered. Indeed, for the first time, the QRPA solution with an exact consideration of the PEP is presented.

The paper is organized as follows. In Sec. II, we describe the solvable model and specify the corresponding solution. Section III describes the standard QRPA and RQRPA within the chosen solvable model. In addition, new extensions of the standard QRPA approach, which take into account the PEP in an approximate way and exactly are introduced, respectively. In Sec. IV, the results obtained within the QRPA approaches are presented and compared with the exact results. Finally in Sec. V, we summarize the results and draw 
some conclusions.

\section{NUCLEAR HAMILTONIAN}

We assume a model Hamiltonian which includes a single-particle term, proton-proton and neutron-neutron pairing and a charge-dependent two-body interaction with particlehole and particle-particle channels included:

$$
H=H_{p}+H_{n}+H_{r e s}
$$

where

$$
\begin{aligned}
H_{\tau} & =e_{\tau} \sum_{m} a_{\tau m}^{\dagger} a_{\tau m}-G_{\tau} S_{\tau}^{\dagger} S_{\tau} \quad(\tau=p, n), \\
H_{r e s} & =2 \chi \beta^{-} \beta^{+}-2 \kappa P^{-} P^{+},
\end{aligned}
$$

with

$$
\begin{aligned}
S_{\tau}^{\dagger} & =\frac{1}{2} \sum_{m} a_{\tau m}^{\dagger} \tilde{a}_{\tau m}^{\dagger}, \\
\beta^{-} & =\sum_{m} a_{p m}^{\dagger} a_{n m}, \quad \beta^{+}=\left(\beta^{-}\right)^{\dagger}, \\
P^{-} & =\sum_{m} a_{p m}^{\dagger} \tilde{a}_{n m}^{\dagger}, \quad P^{+}=\left(P^{-}\right)^{\dagger},
\end{aligned}
$$

$a^{\dagger}(a)$ being the particle creation (annihilation) operator and $\sim$ indicating the time reversed states $\tilde{a}_{\tau m}^{\dagger}=(-1)^{j_{\tau}-m} a_{\tau-m}^{\dagger}$.

The schematic Hamiltonian, given by Eqs. (2.1)-(2.3), reproduces well the QRPA results of the realistic Hamiltonian containing G-matrix elements of the Bonn-OBEP potential for the beta and double beta decay transitions 32 34. The strength $\chi(\kappa)$ of the particle-hole (particle-particle) interaction corresponds to the well-known parameter $g_{p h}\left(g_{p p}\right)$ commonly used in literature [1] to parameterize the realistic ph (pp) interaction.

Performing the Bogolyubov transformation for protons $(\tau=p)$ and neutrons $(\tau=n)$

$$
\alpha_{\tau m}^{\dagger}=u_{\tau} a_{\tau m}^{\dagger}-v_{\tau} \tilde{a}_{\tau m}, \quad \tilde{\alpha}_{\tau m}=v_{\tau} a_{\tau m}^{\dagger}+u_{\tau} \tilde{a}_{\tau m}
$$


which defines the quasiparticle representation, and neglecting the scattering terms $\alpha_{p}^{\dagger} \alpha_{n}$ and $\alpha_{n}^{\dagger} \alpha_{p}$, the model Hamiltonian acquires the form

$$
H_{F}=\epsilon C+\lambda_{1} A^{\dagger} A+\lambda_{2}\left(A^{\dagger} A^{\dagger}+A A\right)
$$

with

$$
\begin{aligned}
C & =\sum_{m} \alpha_{p m}^{\dagger} \alpha_{p m}+\sum_{m} \alpha_{n m}^{\dagger} \alpha_{n m}, \quad A^{\dagger}=\left[\alpha_{p}^{\dagger} \alpha_{n}^{\dagger}\right]^{J=0}, \\
\lambda_{1} & =4 \Omega\left[\chi\left(u_{p}^{2} v_{n}^{2}+v_{p}^{2} u_{n}^{2}\right)-\kappa\left(u_{p}^{2} u_{n}^{2}+v_{p}^{2} v_{n}^{2}\right)\right] \\
\lambda_{2} & =4 \Omega(\chi+\kappa) u_{p} v_{p} u_{n} v_{n} .
\end{aligned}
$$

For the sake of simplicity we used a single level case $j_{p}=j_{n} \equiv j$ and $G_{p}=G_{n} \equiv G$ which implies equal energies for protons and neutrons quasiparticles: $\epsilon=\epsilon_{p}=\epsilon_{n}=\Omega G / 2$ and $v_{i}=\sqrt{N_{i} / 2 \Omega}, u_{i}=\sqrt{1-N_{i} / 2 \Omega}$ with $i=p, n\left(N_{p}\right.$ and $N_{n}$ are number of protons and neutrons, respectively). $\Omega$ denotes the semi-degeneracy of the considered single level.

The model Hamiltonian in Eqs. (2.5) and (2.6), resembles the Hamiltonian of the Lipkin model [19], when $\lambda_{1}$ is taken equal to zero. We note that operators $\left\{A, A^{\dagger}, C\right\}$ are generators for an $\mathrm{SU}(2)$ algebra. Indeed their mutual commutators are:

$$
\left[A, A^{\dagger}\right]=1-\frac{C}{2 \Omega}, \quad\left[C, A^{\dagger}\right]=2 A^{\dagger}, \quad[A, C]=2 A
$$

This model Hamiltonian is expected to account qualitatively for some features of realistic pn-QRPA calculations. Due to these expectations, it has been used to study the standard QRPA, renormalized QRPA as well as the higher order QRPA approximations for the manybody system, Refs. [17,22]. The salient feature of this Hamiltonian is that the stability of the approximate solutions can be discussed in comparison with the exact solution determined by diagonalizing $H_{F}$ in the space of states

$$
\left|n>=\left(A^{+}\right)^{n}\right| 0>, \quad 0 \leq n \leq 2 \Omega .
$$

Here $\mid 0>$ denotes the vacuum state for the quasiparticle operators. The matrix to be diagonalized can be easily calculated with the result: 


$$
\begin{aligned}
<n\left|H_{F}\right| n> & =2 \epsilon n m_{n}+\lambda_{1}\left(m_{n+1}-m_{n}+\frac{n m_{n}}{\Omega}\right), \\
<n-2\left|H_{F}\right| n> & =\lambda_{2} m_{n},
\end{aligned}
$$

where

$$
m_{n} \equiv<0\left|A^{n}\left(A^{\dagger}\right)^{n}\right| 0>=\frac{n !(2 \Omega) !}{(2 \Omega-n) !(2 \Omega)^{n}} \quad(n \leq 2 \Omega) .
$$

For $n>2 \Omega$, the norm overlaps $m_{n}$ are vanishing.

\section{QUASIPARTICLE RANDOM PHASE APPROXIMATION}

Another way to find an excited state for the model Hamiltonian (2.5), is to solve the corresponding QRPA equation. In what follows we shall briefly describe the basic ideas underlying this method for our solvable model.

Within the QRPA, an excited state $\mid Q>$ is created by applying a phonon creation operator $Q^{\dagger}$ on a state $\mid r p a>$ having the properties:

$$
\left|Q>=Q^{\dagger}\right| r p a>, \quad Q \mid r p a>=0 .
$$

The simplest form for the phonon operator, in the fermionic space, is

$$
Q^{\dagger}=X A^{\dagger}-Y A
$$

where $\mathrm{X}$ and $\mathrm{Y}$ are called forward- and backward- going free variational amplitudes and satisfy the QRPA equation:

$$
\left(\begin{array}{ll}
\mathcal{A} & \mathcal{B} \\
\mathcal{B} & \mathcal{A}
\end{array}\right)\left(\begin{array}{l}
X \\
Y
\end{array}\right)=\mathcal{E}_{Q R P A}\left(\begin{array}{cc}
\mathcal{U} & 0 \\
0 & -\mathcal{U}
\end{array}\right)\left(\begin{array}{l}
X \\
Y
\end{array}\right),
$$

where

$$
\begin{aligned}
& \mathcal{A}=<r p a\left|\left[A,\left[H_{F}, A^{\dagger}\right]\right]\right| r p a>, \\
& \mathcal{B}=-<r p a\left|\left[A,\left[H_{F}, A\right]\right]\right| r p a>, \\
& \mathcal{U}=<\operatorname{rpa}\left|\left[A, A^{\dagger}\right]\right| r p a>.
\end{aligned}
$$


It is useful to introduce the notation:

$$
\begin{gathered}
\bar{X}=\mathcal{U}^{1 / 2} X, \quad \bar{Y}=\mathcal{U}^{1 / 2} Y, \\
\overline{\mathcal{A}}=\mathcal{U}^{-1 / 2} \mathcal{A U}^{-1 / 2}, \quad \overline{\mathcal{B}}=\mathcal{U}^{-1 / 2} \mathcal{B U}^{-1 / 2} .
\end{gathered}
$$

Then the QRPA eigenenergy $\mathcal{E}_{Q R P A}$ and the new amplitudes $\bar{X}$ and $\bar{Y}$ are given by:

$$
\begin{aligned}
\mathcal{E}_{Q R P A} & =\left(\overline{\mathcal{A}}^{2}-\overline{\mathcal{B}}^{2}\right)^{1 / 2}, \\
\bar{X} & =\frac{\overline{\mathcal{A}}+\mathcal{E}_{Q R P A}}{{\sqrt{\left(\overline{\mathcal{A}}+\mathcal{E}_{Q R P A}\right)^{2}-\overline{\mathcal{B}}^{2}}}^{2}} \\
\bar{Y} & =\frac{-\overline{\mathcal{B}}}{\sqrt{\left(\overline{\mathcal{A}}+\mathcal{E}_{Q R P A}\right)^{2}-\overline{\mathcal{B}}^{2}}} .
\end{aligned}
$$

From the definition of the QRPA ground state $\mid r p a>$ (3.1), it follows that elements $\mathcal{A}, \mathcal{B}$ and $\mathcal{U}$ of the QRPA equation are function of the $X$ and $Y$ amplitudes. Due to this fact this non-linear eigenvalue problem could be solved only numerically by an iteration process. The functional dependence of $\mathcal{A}, \mathcal{B}, \mathcal{U}$ on $X$ and $Y$ is specific to the approximation scheme and influences crucially the final results. Below, we shall discuss, separately, several approaches.

The standard QRPA: The simplest approximation scheme to calculate of $\mathcal{A}, \mathcal{B}$ and $\mathcal{U}$ is the quasiboson approximation (QBA), which assumes $\left[A, A^{\dagger}\right] \approx<\left|\left[A, A^{\dagger}\right]\right|>=1$, i.e. $A$ and $A^{\dagger}$ are considered to be boson operators. Here $\mid>$ denotes the uncorrelated BCS ground state. In this case, one finds the expressions:

$$
\mathcal{A}=2 \epsilon+\lambda_{1}, \quad \mathcal{B}=2 \lambda_{2}, \quad \mathcal{U}=1,
$$

which determine the excited state eigenenergy and wavefunction with normalization $X^{2}-$ $Y^{2}=1$. The drawback of this approximation scheme is the collapse of the standard QRPA solution within the physically acceptable interval for the nucleon-nucleon interaction strength.

The renormalized QRPA: The renormalized QRPA (RQRPA) approach avoids the collapse of the QRPA solution for physical parameters of the nuclear Hamiltonian. Within the 
RQRPA the commutator, $\left[A, A^{\dagger}\right]$ is replaced with its expectation value in the ground state $D=<\operatorname{rpa}\left|\left[A, A^{\dagger}\right]\right| r p a>($ renormalized QBA). This modifies the matrices $\mathcal{A}, \mathcal{B}, \mathcal{U}$ in the following way 13,14:

$$
\mathcal{A}=2 \epsilon D+\lambda_{1} D^{2}, \quad \mathcal{B}=2 \lambda_{2} D^{2}, \quad \mathcal{U}=D=\left(1+\frac{\bar{Y}^{2}}{\Omega}\right)^{-1}
$$

Note that the fermionic structure of the $A, A^{\dagger}$ operators is taken into account only in an approximate way. In the limit of $D=1$, i.e. the $\mid r p a>$ ground state is replaced by the BCS one $\mid>$, one gets the standard QRPA approach.

It is worth to remark that in both the standard QRPA and the RQRPA, the elements $\mathcal{A}, \mathcal{B}$ and $\mathcal{U}$ are evaluated by using some approximate schemes for the commutator $\left[A, A^{\dagger}\right]$. If the commutator is exactly considered, i.e. the PEP is fulfilled, the matrices $\mathcal{A}, \mathcal{B}$ and $\mathcal{U}$ take the form:

$$
\begin{gathered}
\mathcal{A}=\left(2 \epsilon+\lambda_{1}\right)-\left(\epsilon+\lambda_{1}\right) \frac{<r p a|C| r p a\rangle}{\Omega}+\lambda_{1} \frac{<r p a|C C| r p a\rangle}{4 \Omega^{2}}- \\
\lambda_{1} \frac{<r p a\left|A^{\dagger} A\right| r p a>}{\Omega}-2 \lambda_{2} \frac{<r p a\left|A^{\dagger} A^{\dagger}\right| r p a>}{\Omega}, \\
\mathcal{B}=\lambda_{2}\left(2-\frac{1}{\Omega}\right)-\lambda_{2}\left(2-\frac{1}{2 \Omega}\right) \frac{<r p a|C| r p a>}{\Omega}+\lambda_{2} \frac{<r p a|C C| r p a>}{2 \Omega^{2}}- \\
2 \lambda_{2} \frac{<r p a\left|A^{\dagger} A\right| r p a>}{\Omega}-\lambda_{1} \frac{<r p a|A A| r p a>}{\Omega}, \\
\mathcal{U}=1-\frac{<r p a|C| r p a>}{2 \Omega} .
\end{gathered}
$$

The calculation of the involved matrix elements requires the knowledge of the $\mid r p a>$ ground state, determined by the condition in Eq. (3.1). The analytical form for $\mid r p a>$ is known within QBA and renormalized QBA. For the phonon operator given by the Eq. (3.2), one obtains:

$$
\left|r p a>_{Q B A}=n e^{-d A^{\dagger} A^{\dagger}}\right|>, \quad d=-\frac{Y}{2 X}
$$

where $n$ stands for the normalization factor. 
In general, it is not possible to find an explicit expression for $\mid r p a>$ unless some additional approximation is adopted. Fortunately, this can be achieved in the case of the solvable model considered in the present paper. By solving the Eq. (3.1), one obtainst]

$$
\left|r p a>_{e x c .}=N \sum_{0}^{\Omega} \beta_{l}\left(\frac{Y}{X}\right)^{l}\left(A^{\dagger} A^{\dagger}\right)^{l}\right|>,
$$

with

$$
\beta_{l}=(2 \Omega)^{l} \frac{\Omega !}{(2 \Omega) !} \frac{(2 \Omega-2 l) !}{l !(\Omega-l) !}, \quad N^{-2}=\sum_{0}^{\Omega} \beta_{l}^{2}\left(\frac{Y}{X}\right)^{2 l} m_{2 l} .
$$

We note that if the ground state correlations are neglected in the calculation of $\mathcal{A}, \mathcal{B}$ and $\mathcal{U}$ in Eqs. (3.10)-(3.12), i.e. the RPA vacuum $\mid r p a>$ is replaced with BCS vacuum $\mid>$, one gets: $\mathcal{A}=\left(2 \epsilon+\lambda_{1}\right), \mathcal{B}=\lambda_{2}\left(2-\frac{1}{\Omega}\right)$ and $\mathcal{U}=1$. Obviously, the standard QRPA equation is recovered in the limit $\Omega \rightarrow \infty$, i.e. the bifermion operators $A^{+}$and $A$ behave like bosons.

By using the approximate $|r p a\rangle_{Q B A}$ (3.13) and exact $|r p a\rangle_{\text {exc. }}$ (3.15) solutions for the QRPA ground state, one achieves, in fact, new extensions of the standard QRPA approach, namely the QRPA with the PEP (PP QRPA) included in an approximate manner and the QRPA with the PEP (EPP QRPA) fully fulfilled, respectively. Both methods go beyond the renormalized QRPA approach and require to evaluate ground state expectations values of the $C, C C, A^{\dagger} A^{\dagger}$ and $A^{\dagger} A$ operators entering the expression for $\mathcal{A}, \mathcal{B}$ and $\mathcal{U}$ as shown in Eqs. (3.10)-(3.12). This is performed free of any approximation.

The QRPA with PEP: There is an important difference between this method and the RQRPA one, although both methods use the same ground state wave functions [see Eq. (3.12)]. Indeed, within the RQRPA, the commutator of the two bifermion operators $A$ and $A^{\dagger}$ is considered in approximate way while the PP QRPA takes it exactly. Indeed, the operators of interest have the expectation values:

\footnotetext{
${ }^{1}$ After the present paper was completed we learned that this equation has been derived also by other authors 30,31,25]
} 


$$
\begin{aligned}
& { }_{Q B A}<r p a|C| r p a>_{Q B A}=-4 n^{2} d h_{2}(d), \\
& { }_{Q B A}<r p a|C C| r p a>_{Q B A}=-16 n^{2}\left(d h_{2}(d)-d^{2} h_{4}(d)\right), \\
& { }_{Q B A}<r p a\left|A^{\dagger} A^{\dagger}\right| r p a>_{Q B A}=n^{2} h_{2}(d), \\
& { }_{Q B A}<r p a\left|A^{\dagger} A\right| r p a>_{Q B A}=-n^{2}\left(\left(2-\frac{1}{\Omega}\right) d h_{2}(d)+2 \frac{d^{2}}{\Omega} h_{4}(d)\right) .
\end{aligned}
$$

where the following notations have been used:

$$
\begin{aligned}
h_{0}(d) \equiv & <\left|e^{-d A A} e^{-d A^{\dagger} A^{\dagger}}\right|>=\frac{1}{n^{2}}, \\
& =\sum_{j=0}^{\Omega} \frac{d^{2 j}}{(j !)^{2}} m_{2 j} \approx m_{0}+d^{2} m_{2}+\frac{d^{4}}{4} m_{4}, \\
h_{2}(d) \equiv< & \left|e^{-d A A} A^{\dagger} A^{\dagger} e^{-d A^{\dagger} A^{\dagger}}\right|>, \\
= & \sum_{j=0}^{\Omega-1} \frac{d^{2 j}}{(j !)^{2}} \frac{-d}{j+1} m_{2 j+2} \approx-d m_{2}-\frac{d^{3}}{2} m_{4}, \\
h_{4}(d) \equiv< & \mid e^{-d A A}\left(A^{\dagger}\right)^{4} e^{-d A^{\dagger} A^{\dagger} \mid>,} \\
= & \sum_{j=0}^{\Omega-2} \frac{d^{2 j}}{(j !)^{2}} \frac{d^{2}}{(j+2)(j+1)} m_{2 j+4} \approx \frac{d^{2}}{2} m_{4},
\end{aligned}
$$

We hope that this approach can be applied also for realistic calculations and within a large model space. Note that knowing $\mid r p a>_{Q B A}$, the QRPA matrices can be evaluated without the PEP violation, at least perturbatively with respect to the factor $d$. If the pertubative series is truncated to the quadratic terms in $\mathrm{d}$, the resulting approach will be hereafter labeled by the abbreviation PP2 QRPA.

The QRPA with exact PEP: This method can be formulated only for a solvable model for which the exact QRPA ground state can be analytically found. Within the EPP QRPA there is no violation of the PEP. However, one can not expect that the EPP QRPA solution coincides with the exact solution for the first excited state of the nuclear Hamiltonian (2.5). The difference is caused by the approximations incorporated in the construction of the operator $Q^{\dagger}$ determining the excited state [see Eq. (3.1)]. Therefore, from the direct comparison with the exact solution one may conclude how far the approximate description, 
with the phonon operator of a simple structure, is from the exact picture (3.1). The following expressions are used in elaborating the above defined procedure:

$$
\begin{aligned}
& { }_{\text {exc. }}<r p a|A A| r p a>_{\text {exc. }}={ }_{e x c .}<r p a\left|A^{\dagger} A^{\dagger}\right| r p a>_{e x c .}=N^{2} \sum_{l=0}^{\Omega-1} \beta_{l} \beta_{l+1}\left(\frac{Y}{X}\right)^{2 l+1} m_{2 l+2}, \\
& { }_{\text {exc. }}<r p a\left|A A^{\dagger}\right| r p a>_{\text {exc. }}=N^{2} \sum_{l=0}^{\Omega-1} \beta_{l}^{2}\left(\frac{Y}{X}\right)^{2 l} m_{2 l+1} \\
& { }_{e x c .}<r p a|C| r p a>_{e x c .}=N^{2} \sum_{l=1}^{\Omega} \beta_{l}^{2}(4 l)\left(\frac{Y}{X}\right)^{2 l} m_{2 l} \\
& { }_{\text {exc. }}<r p a|C C| r p a>_{\text {exc. }}=N^{2} \sum_{l=1}^{\Omega} \beta_{l}^{2}(4 l)^{2}\left(\frac{Y}{X}\right)^{2 l} m_{2 l}, \\
& { }_{e x c .}<r p a\left|A^{\dagger} A\right| r p a>_{e x c .}=-1+\frac{e x c .}{<r p a|C| r p a>_{e x c .}}+_{e x c .}<r p a\left|A A^{\dagger}\right| r p a>_{e x c .} .
\end{aligned}
$$

\section{RESULTS AND DISCUSSIONS}

In what follow we shall present the numerical results for the QRPA approaches described in the previous section and compare them with the values provided by diagonalizing $H_{F}(2.5)$. In order to continue and complete the discussion of this Hamiltonian given in Refs. [17,22, we have chosen the same set of parameters as there:

$$
j=9 / 2, \quad Z=4, \quad N=6, \quad e=1 M e V,
$$

which determine the BCS amplitudes entering the lambda $a_{1,2}$ parameters of the model Hamiltonian $H_{F}$. Also we redefine the parameters $\kappa$ and $\chi$ as in Refs. [17,22]:

$$
\kappa \rightarrow \kappa^{\prime} \equiv 2 \Omega \kappa, \quad \chi \rightarrow \chi^{\prime} \equiv 2 \Omega \chi
$$

The values $\chi^{\prime}=0$. and $\chi^{\prime}=0.5$ were adopted while the particle-particle strength $\kappa^{\prime}$ was

allowed to vary in the interval $0 \leq \kappa^{\prime} \leq 2$. Comparing the schematic calculations with the realistic ones, a value for $\kappa^{\prime}$ close to unity is expected. 


\section{A. Excitation energies}

In Fig. 1 1 we plotted the dependence of the QRPA excitation energy and the first excitation energy obtained by diagonalizing $H_{F}$ (bold solid line) on $\kappa^{\prime}$, for $\chi^{\prime}=0$ (upper figure) and $\chi^{\prime}=0.5$ (lower figure), respectively. Note that the standard QRPA breaks down for $\kappa^{\prime} \approx 1$. The RQRPA excitation energy remains real within the whole interval of $\kappa^{\prime}$, although it deviates significantly from the exact solution beyond the breaking down point of the standard QRPA. The effect is more evident for $\chi^{\prime}=0.5$. The PP2 QRPA and, especially, the PP QRPA energies reproduce quite well those of $H_{F}$, except for the values of $\kappa^{\prime}$ approaching their minimum. The EPP QRPA, which take into account the PEP exactly, systematically overestimates the results obtained through the diagonalization of $H_{F}$. This difference might be attributed to the simple form of the phonon operator $Q^{\dagger}$ (3.2). From this figure one remarks that the collapse is shifted to a large value of $\kappa^{\prime}$ when the PEP is satisfied to a larger extent. For example for the PP2 QRPA, PP QRPA and EPP QRPA, the collapse appear at about $\kappa^{\prime}=1.80, \kappa^{\prime}=2.50$ and $\kappa^{\prime}=2.55\left(\chi^{\prime}=0.5\right)$, respectively. This indicates that a real phase transition could take place in the region beyond $\kappa^{\prime}=2.5$.

\section{B. Expectation values of the quasiparticle number operator}

In order to get additional information about the quality of different approximations, we

calculate the expectation values of the quasiparticle number operator in the ground and first excited states. These are defined as follows:

$$
N_{0} \equiv<r p a\left|\frac{C}{2}\right| r p a>, \quad \Delta N \equiv<r p a\left|Q \frac{C}{2} Q^{\dagger}\right| r p a>-N_{0} .
$$

For the situations defined before, the results are:

$$
\begin{aligned}
N_{0} & =Y^{2}, & (Q R P A), \\
& =\bar{Y}^{2}, & (R Q R P A), \\
& =\frac{2 d^{2} m_{2}}{1+d^{2} m_{2}}, & (P P 2 Q R P A)
\end{aligned}
$$




$$
\begin{aligned}
& =n^{2}(-2 d) h_{2}(d), \quad(P P Q R P A) \\
& ={ }_{\text {exc. }}<r p a|C| r p a>_{\text {exc. }}, \quad(\operatorname{EPP} Q R P A) \\
& \Delta N=1+2 Y^{2}, \quad(Q R P A), \\
& =1+2 \bar{Y}^{2}, \quad(R Q R P A), \\
& =\left(X^{2}+Y^{2}\right)+\left(X^{2}-Y^{2}-1-\frac{X^{2}+Y^{2}}{\Omega}\right) \frac{Q B A}{<r p a|C| r p a>_{Q B A}}- \\
& {\frac{X^{2}-Y^{2}}{4 \Omega}}_{Q B A}<r p a|C C| r p a>_{Q B A} \quad(P P Q R P A),
\end{aligned}
$$

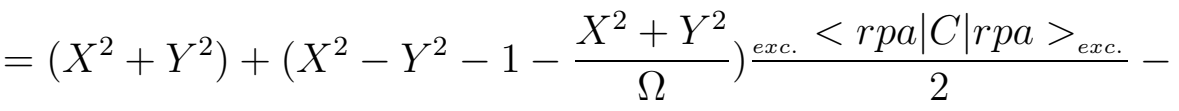

$$
\begin{aligned}
& {\frac{X^{2}-Y^{2}}{4 \Omega}}_{e x c .}<r p a|C C| r p a>_{e x c .} \quad(\operatorname{EPP} Q R P A) .
\end{aligned}
$$

The expressions corresponding to the standard QRPA and RQRPA have been obtained by replacing the operator $C / 2$ with its boson image, respectively. As for the remaining case we stay in the fermionic space and use the commutation algebra given by the Eq. (2.7).

In Figs. 2 and 3, $N_{0}$ and $\Delta N$, given by the above listed approximation schemes as well as the exact calculation are presented. One sees that the standard QRPA overestimates the ground state correlations near the collapse point, which reflects itself in a sudden increase of the average quasiparticle number. The RQRPA does not collapse at all and overestimates the exact result both for $N_{0}$ and $\Delta N$. A distinct situation is produced by the QRPA approaches with PEP. There occurs an underestimation of the ground state correlations and the values of $N_{0}$ are smaller than those corresponding to the exact solution. Practically, there is no difference between the PP2 QRPA and the PP QRPA up to the point where the former one collapses. The EPP QRPA, with an exact treatment of the PEP, provides the best agreement with the exact results obtained by diagonalizing $H_{F}$. We notice that none of the considered QRPA methods is able to reproduce the exact result for $\Delta N$ which, after a certain point in the region $\kappa^{\prime} \doteq 1 .-1.1$, is falling down, contrary to the behavior of other curves [22]. This is an indication that a more complex form for the phonon operator might be necessary. 


\section{Fermi $\beta$ transition amplitudes}

We turn our attention now to transitions induced by the Fermi $\beta^{ \pm}$operators. Neglecting the scattering term, as we did for the nuclear Hamiltonian $H_{F}$ (see Sec. II), the Fermi $\beta^{ \pm}$ operators, in the quasiparticle basis, take the form

$$
\beta^{-}=\sqrt{2 \Omega}\left(u_{p} v_{n} A^{\dagger}+v_{p} u_{n} A\right), \quad \beta^{+}=\left(\beta^{-}\right)^{\dagger}
$$

The matrix elements of $\beta^{-}$operator between ground and first excited states corresponding to different versions of the QRPA, are given as follows:

$$
\begin{aligned}
<0_{1}^{+}\left|\beta^{-}\right| r p a> & =\sqrt{2 \Omega}\left(X u_{p} v_{n}+Y v_{p} u_{n}\right) \quad(Q R P A), \\
& =\sqrt{2 \Omega}\left(\bar{X} u_{p} v_{n}+\bar{Y} v_{p} u_{n}\right) D^{1 / 2} \quad(R Q R P A), \\
& =\sqrt{2 \Omega}\left(X u_{p} v_{n}+Y v_{p} u_{n}\right) \frac{1+d^{2}\left(1-\frac{2}{\Omega}\right) m_{2}}{1+d^{2} m_{2}} \quad(P P 2 Q R P A), \\
& =\sqrt{2 \Omega} n^{2}\left(X u_{p} v_{n}+Y v_{p} u_{n}\right)\left(h_{0}(d)+2 \frac{d}{\Omega} h_{2}(d)\right) \quad(P P Q R P A), \\
= & \sqrt{2 \Omega}\left(X u_{p} v_{n}+Y v_{p} u_{n}\right) \times \\
& \quad\left(1-\frac{e x c .}{<r p a|C| r p a>_{e x c .}}\right) \quad(E P P Q R P A) .
\end{aligned}
$$

Expressions for $\beta^{+}$are obtainable from the above equations by interchanging the $u^{\prime}$ s and $v^{\prime} \mathrm{s}$.

In Figs. 4 and 5 we examine the behavior of the $\beta^{-}$and $\beta^{+}$amplitudes respectively as functions of $\kappa^{\prime}$ for two values for $\chi^{\prime}(=0 ., 0.5)$. One notes a rapid increase of $\beta^{-}$and decrease of $\beta^{+}$transition strengths for the standard QRPA and the PP2 QRPA, close to their collapse point. In general, the PP2 QRPA, the PP QRPA and the EPP QRPA methods reproduce better the trends of the exact results comparing them with the RQRPA. The best agreement with the exact values is achieved for the PP QRPA. Obviously these analysis demonstrate how important it is to have a correct treatment of the PEP, in describing the nuclear $\beta$ transitions. It is worth noting the sensitivity of the $\beta^{+}$amplitude to the details of nuclear structure wave functions. Indeed, by increasing the the particle-particle interaction 
strength $\kappa$, the matrix element of the $\beta^{+}$transition operator reaches a vanishing value and therefore is no longer predictable.

\section{The Ikeda sum rule}

From the $\beta^{ \pm}$amplitudes one obtains, straightforwardly, the $\beta^{ \pm}$strengths:

$$
S^{-}=\left|<0_{1}^{+}\right| \beta^{-}|r p a>|^{2}, \quad S^{+}=\left|<0_{1}^{+}\right| \beta^{+}|r p a>|^{2} .
$$

For a ground state preserving the proton and neutron numbers in average, the two strengths are satisfying the Ikeda sum rule

$$
S^{-}-S^{+}=N-Z
$$

where $\mathrm{N}$ and $\mathrm{Z}$ are the numbers of neutrons and protons, respectively.

It is well known that the Ikeda Sum rule is automatically fullfiled in the standard QRPA and violated in the RQRPA [16,17]. According to the Fig. 6, this is also true for the solvable model of this work. This figure shows also numerical results for the Ikeda sum rule predicted by the methods described so far, and compare them with the exact results. One notices that the exact results for $H_{F}$, do not fulfill the Ikeda sum rule and show a large deviation from the value of $(\mathrm{N}-\mathrm{Z})$ for $\kappa^{\prime}$ for $\kappa^{\prime} \geq 1$. The origin of this phenomenon is expected to due to neglecting the scattering terms in the derivation of the assumed Hamiltonian $H_{F}$ [35]. We hope that discrepancies concerning the Ikeda sum rule are substantially diminished by adding the contributions due to the quasiparticle operators $a_{\tau}^{\dagger} a_{\tau}, a_{\tau}^{\dagger} a_{\tau}^{\dagger}+a_{\tau} a_{\tau}$ involved in the particle number operators. Indeed these contributions have been omitted so far, although their average on the ground state is not vanishing. One may conclude that the standard QRPA fails to reproduce the exact results for the chosen Hamiltonian. All other modifications of the QRPA (RQRPA, PP2 QRPA, PP QRPA, EPP QRPA) reproduce the trend of the exact solution, albeit the agreement with the exact results, for large value of $\kappa^{\prime}$ $\left(\kappa^{\prime} \geq 1\right)$ is rather poor. For non-vanishing $\kappa^{\prime}$ the results lying closest to the exact ones are 
those produced by PP QRPA and FPP QRPA. A reason might be the influence of higher excited states on the ground state induced by the diagonalization procedure. It is worthwhile to notice that improving the treatment of the PEP in the QRPA, a better agreement of the Ikeda sum rule with the $(\mathrm{N}-\mathrm{Z})$ value is achieved.

\section{E. Double beta decay matrix element}

In this section we shall focus our attention on the two-neutrino double beta decay mode, $2 \nu \beta \beta$. Consequences of the previously presented approaches on the $2 \nu \beta \beta$-decay matrix element will be discussed. Within the solvable model considered here there is only one QRPA excited state and the corresponding nuclear $2 \nu \beta \beta$-decay matrix element takes the form

$$
M_{F}^{2 \nu}=\frac{f<r p a\left|\beta^{-}\right| 0_{1}^{+}>_{f i}<0_{1}^{+}\left|\beta^{-}\right| r p a>_{i}}{\mathcal{E}_{Q R P A}+\Delta} .
$$

Here, the states with subscripts "i" and "f" are describing to the initial (A,Z) and final $(\mathrm{A}, \mathrm{Z}+2)$ nuclei, respectively. We considered $\Delta$ to be equal to $0.5 \mathrm{MeV}$ and performed the calculations for the following set of parameters [17]:

$$
j=19 / 2(Z=6, \quad N=14) \rightarrow(Z+2=8, \quad N-2=12), \quad \chi^{\prime}=0,0.5
$$

Results corresponding to the matrix element $M_{F}^{2 \nu}$, calculated with different approximations, are shown in Fig. 0, as function of $\kappa^{\prime}$. In addition we present also the exact results of $H_{F}$, considering only the contribution coming from the lowest intermediate state. One notices that the behaviors of the QRPA and the RQRPA curves are qualitatively similar to those found in the realistic calculations. The transition amplitude $M_{F}^{2 \nu}$ vanishes within the range $1.0 \leq \kappa^{\prime} \leq 1.5$ for all calculations, including the exact ones. The values of $M_{F}^{2 \nu}$ obtained by a better consideration of the Pauli exclusion principle (PP QRPA and EPP QRPA) are significantly less sensitive to the particle-particle interaction strength $\kappa^{\prime}$. Our studies show that the behavior of $M_{F}^{2 \nu}$, as function of $\kappa^{\prime}$, is strongly influenced by the $\beta^{+}$ strength characterizing final nucleus. 


\section{SUMMARY AND CONCLUSIONS}

We have analyzed some limitations of the QRPA formalism in an solvable proton-neutron monopole Lipkin model. In addition to the standard QRPA and RQRPA, we introduced new extensions of the standard QRPA approach by improving (PP QRPA) and exactly (EPP QRPA) considering the Pauli exclusion principlę These approaches have been used to study the behavior of different observables as function of the particle-particle interaction strength $\kappa^{\prime}$.

Due to the collapse of its solution for $\kappa^{\prime} \approx 1.0$, the standard QRPA reproduces worse the exact results of the nuclear Hamiltonian. Our studies show that the real collapse of the QRPA, usually associated to a phase transition, appears for a large value of $\kappa^{\prime}(\approx 2.5)$. For the PP2 QRPA, which is obtained from the PP QRPA by cutting the series in $d$ at second order, the solution breakdown appears around $\kappa^{\prime}=1.7$. This suggests that a better consideration of the PEP, within the QRPA, shifts the instability strength to a larger value.

To shed more light on this problem we commented on the corrections induced by the ground state correlations, by plotting the average quasiparticle number versus $\kappa^{\prime}$. Our analysis of the $\beta^{ \pm}$transitions shows the sensitivity of the $\beta^{+}$transition to correlations, included in the ground state, which violate the PEP. Concerning the Ikeda sum rule we have found that this is conserved neither by the exact solution of the quasiparticle nuclear Hamiltonian nor by the QRPA methods including the PEP. This discrepancy is very likely due to the structure of the model Hamiltonian. Also one expects, that including the contribution coming from the two quasiparticle and quasiparticle scattering operators entering the expressions of the particle number operators, the discrepancies are decreased.

\footnotetext{
${ }^{2}$ After the present work was completed we were aware about a certain overlap of the method EPP QRPA presented here and the self-consistent QRPA approach proposed in Ref. 31. However there are also important differences such as i) the $u$ and v coefficients are determined differently ii) the model Hamiltonians are different iii) our papers have distinct objectives.
} 
The $2 \nu \beta \beta$-decay matrix element, calculated within the solvable model, is changing the sign when $\kappa^{\prime}$ is increased, similar to what happens in realistic calculations. It was pointed out that $M_{F}^{2 \nu}$ calculated by the PP QRPA and EPP QRPA, is less sensitive to the details of nuclear structure than that predicted by the standard QRPA and RQRPA approaches.

In discussing the results of the PP2 QRPA, PP QRPA and EPP QRPA we should keep in mind that the first two methods are just approximations of the last method. As expected, the PP QRPA reproduces the EPP QRPA solution better than the PP2 QRPA since it uses a complete pertubative expansion. The difference between the EPP QRPA and exact solution originates in the simple form of the phonon operator which ignores nonlinear terms like for example those proportional to the $A^{+} A^{+} A$ and $A^{+} A A$ operators. To our knowledge, we are the first showing the inaccuracy coming exclusively from a simple form of the widely used phonon operator in the QRPA. An interesting point is the connection of the EPP QRPA and the PP QRPA to the exact solution. We note that there is no principle reason which would determine that the EPP QRPA results should reproduce the exact ones better than the PP QRPA. It turns out that the combined effect coming from the simple form of the phonon operator and the simple RPA wave functions, derived within quasiboson approximations, leads to a better agreement with exact solution for PP QRPA in some cases, e.g. for the lowest excitation energy (Fig. 1) and $\beta^{+}$transition amplitudes (Fig. 5) and worse for other observables. For example the EPP QRPA reproduces the exact results better than PP QRPA when the expectation values of the quasiparticle number operators (Figs. 8 and 3 ) and Ikeda Sum Rule (Fig. 6) are calculated.

The main conclusions of our analysis can be summarized as follows.

New extensions of the standard QRPA with approximate (PP QRPA) and exact (EPP QRPA) consideration of the PEP were presented. These formalisms yield a better agreement with the exact results, obtained by diagonalizing the model Hamiltonian, than the standard QRPA and the renormalized QRPA approaches.

The EPP QRPA results show that the collapse of the first excited states is far from the place where the standard QRPA breaks down, i.e., is achieved for larger values of the 
particle-particle interaction strength.

The comparison of the EPP QRPA results with those obtained with the exact eigenstates of $H_{F}$, points out the drawbacks coming from the simple structure of the QRPA phonon operator and suggests a range of applicability for this theory. Clearly, this analysis shows some limitations for the QRPA and RQRPA approaches.

The results of the present paper support our hope that the PP QRPA approximations might work equally well in the case of realistic calculations for large model space, which are expected to offer more reliable results for the double beta decay transitions than the standard QRPA and the RQRPA ones. Indeed, the PP QRPA is based on the approximate QRPA ground state wave functions, derived within the QBA, which can be undoubtly found also in realistic models. This subject seems to be very interesting and therefore deserves further considerations. We intend to apply the PP2 QRPA method first for a realistic models with separable forces 32 .

\section{ACKNOWLEDGMENTS}

We acknowledge a partial support from the CNSIS under the contract 39C (A. A. R.), the Deutsche Forschungsgemeinschaft Fa67/17-1 and Fa67/19-1 and the Grant Agency of the Czech Republic grant No. 202/98/1216 (F. ̌̌s). 


\section{REFERENCES}

[1] P. Vogel and M.R. Zirnbauer, Phys. Rev. Lett. 57, 731 (1986).

[2] J. Engel, P. Vogel, and M. R. Zirnbauer, Phys. Rev. C 37, 731 (1988).

[3] O. Civitarese, A. Faessler, and T. Tomoda, Phys. Lett. B 194, 11 (1987).

[4] K. Muto, E. Bender, and H. V. Klapdor, Z. Phys. A 334, 177 (1989).

[5] J. Suhonen, T. Taigel, and A. Faessler, Nucl. Phys. A 591, 195 (1995).

[6] A. A. Raduta, A. Faessler, S. Stoica, and W. A. Kamiński, Phys. Lett. B 254, 7 (1991), Nucl. Phys. 534, 149 (1991).

[7] M.K. Cheon, A. Bobyk, A. Faessler, F. Šimkovic, and G. Teneva, Nucl. Phys. A 561, 74 (1993); A 564, 329 (1993).

[8] J. Hirsch and F. Krmpotić, Phys. Rev. C 41, 792 (1990).

[9] F. Krmpotić, Phys. Rev. C 48, 1452 (1993).

[10] G. Pantis, F. Šimkovic, J.D. Vergados, A. Faessler, Phys. Rev. C 53, 695 (1996).

[11] D. Karadjov, V.V. Voronov, and F. Catara, Phys. Lett. B 306, 197 (1993).

[12] F. Catara, N. Dinh Dang and M. Sambataro, Nucl. Phys. A 579, 1 (1994).

[13] J. Toivanen and J. Suhonen, Phys. Rev. Lett. 75, 410 (1995).

[14] J. Schwieger, F. Šimkovic, and A. Faessler, Nucl. Phys. A 600, 179 (1996). 782 (1997).

[15] A. Faessler and F. Šimkovic, J. Phys. G 24, 2139 (1998).

[16] F. Krmpotić, T.T.S. Kuo, A. Mariano, E.J.V. de Passos and A.F.R. de Toledo Piza, Nucl. Phys. A 612, 223 (1997); Fizika B5, 93 (1996).

[17] J.G. Hirsch, P.O. Hess and O. Civitarese, Phys. Rev. C 54, 1976 (1996). 
[18] A.A. Raduta, D. Delion, and A. Faessler, Phys. Rev. C 51, 3008 (1995); Nucl. Phys. A 617, 176 (1997).

[19] H.J. Lipkin, N. Meshkov, and S. Glick, Nucl. Phys. A 62, 188 (1965).

[20] S.A. Moszkowski, Phys. Rev. 110, 403 (1958).

[21] J.G. Hirsch, P.O. Hess and O. Civitarese, Phys. Rev. C 56, 199 (1997).

[22] M. Sambataro and J. Suhonen, Phys. Rev. C 56, 782 (1997).

[23] M. Sambataro, Phys. Rev. C 59, 2056 (1999).

[24] J.G. Hirsch, P.O. Hess and O. Civitarese, Phys. Rev. C 60, 064303 (1999).

[25] J.G. Hirsch, O. Civitarese, and M. Reboiro, Phys. Rev. C 60, 024309 (1999).

[26] M. Sambataro and N. Dinh Dang, Phys. Rev. C 59, 1422 (1999).

[27] J. Engel, S. Pittel, M. Stoitsov, P. Vogel, and J. Dukelsky, Phys. Rev. C 55, 1781 (1997).

[28] J. Dobes and S. Pittel, Phys. Rev. C 57, 688 (1998).

[29] E.J.V. de Passos, A.F.R. de Toledo Piza, F. Krmpotić, Phys. Rev. C 58, 1841 (1998)

[30] A. A. Raduta, V. Ceausescu and E. Badralexe, St. Cerc. Fiz. 28, 617 (1976).

[31] F. Krmpotić, E.J.V. de Passos, D.S. Delion, J. Dukelsky, and P. Schuck, Nucl. Phys. A 637, 295 (1998).

[32] V. A. Kuz'min and V. G. Soloviev, Nucl. Phys. A 486, 118 (1988).

[33] O. Civitarese and J. Suhonen, Nucl. Phys. A 578, 62 (1994).

[34] O. Civitarese, J. Suhonen, and A. Faessler, Nucl. Phys. A 591, 195 (1995).

[35] A.A. Raduta, C.M. Raduta, A. Faessler, and W. Kamiński, Nucl. Phys. A 634, 497 (1998). 


\section{FIGURES}

FIG. 1. Comparison of the lowest excitation energies resulting from the diagonalization of $H_{F}$ (bold solid line - $H_{F}$ diag.) with the standard QRPA (solid line - QRPA), the renormalized QRPA (dashed line - RQRPA), the QRPA with the Pauli exlusion principle up to second order in d (bold dashed line - PP2 QRPA), the QRPA with Pauli exclusion principle (bold dotted line - PP QRPA) and the QRPA with exact consideration of Pauli exclusion principle (bold dot-dashed line - EPP QRPA) values.

FIG. 2. The expectation values of (half) the quasiparticle total number operator, $\mathrm{C} / 2$, in the ground state, versus $\kappa^{\prime}$. Conventions are the same as in Fig.1.

FIG. 3. Differences between the expectation values of (half) quasiparticle number operator, $\mathrm{C} / 2$, in the first excited state and in the ground state as function of $\kappa^{\prime}$. Conventions are the same as in Fig.1.

FIG. 4. The Fermi $\beta^{-}$transition amplitudes between the ground and first excited states. Conventions are the same as in Fig.1.

FIG. 5. The Fermi $\beta^{+}$transition amplitudes between the ground and first excited states. Conventions are the same as in Fig.1.

FIG. 6. The Fermi $\beta^{-}$transition amplitudes between the ground and first excited states. Conventions are the same as in Fig.1.

FIG. 7. The $2 \nu \beta \beta$-decay Fermi transition amplitude versus $\kappa^{\prime}$. The same notations as in Fig. 1 are used. 

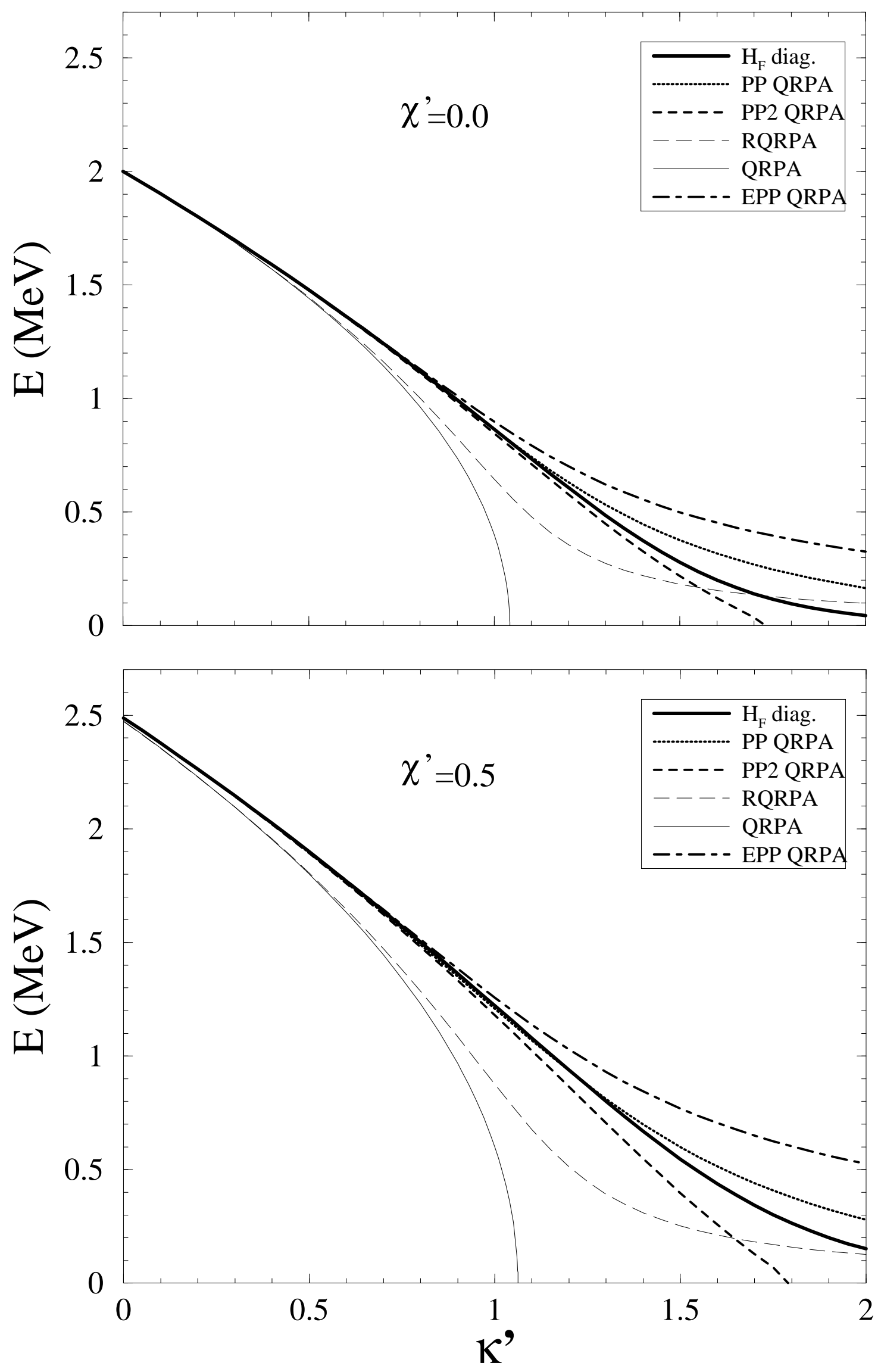

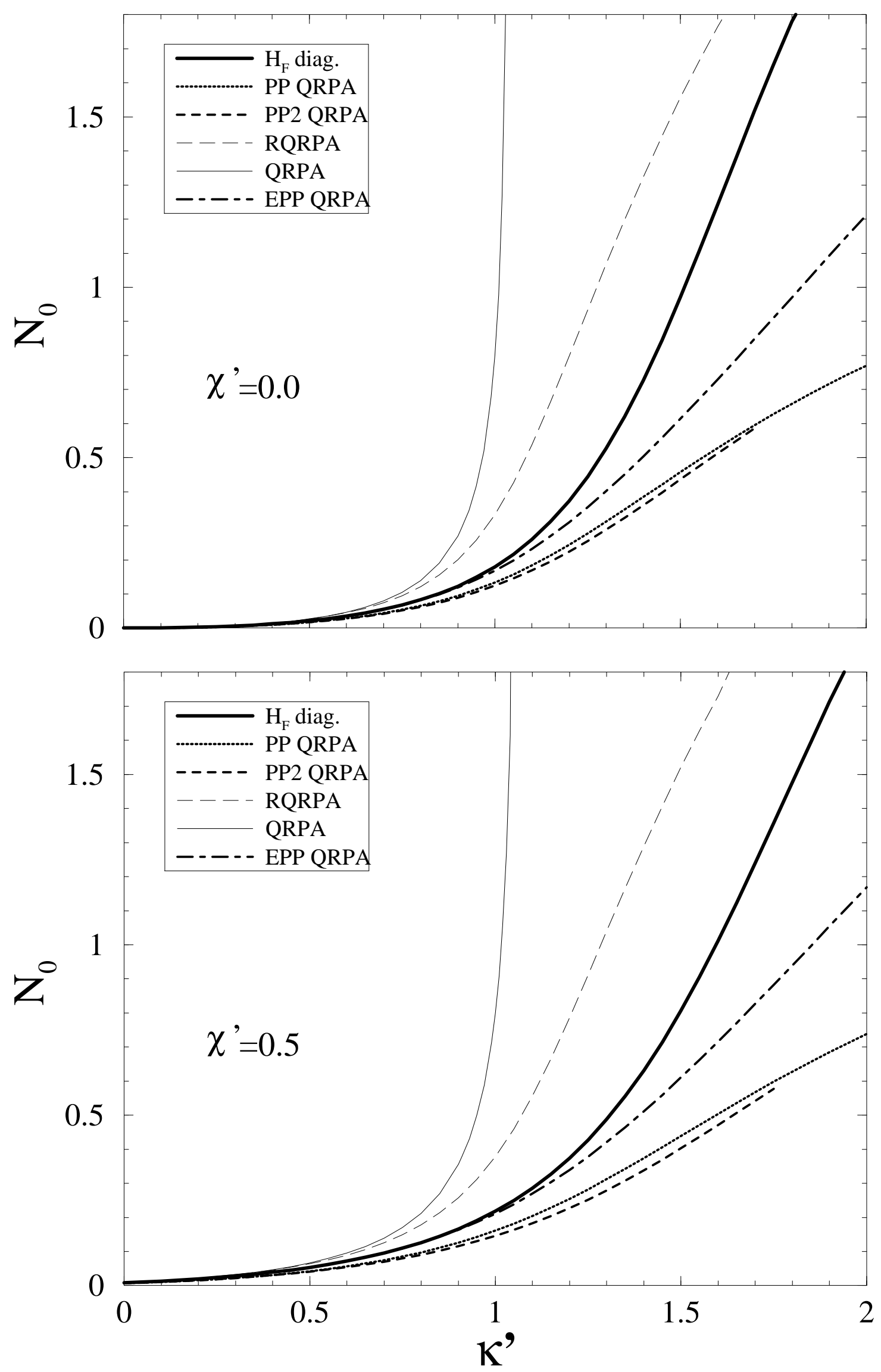

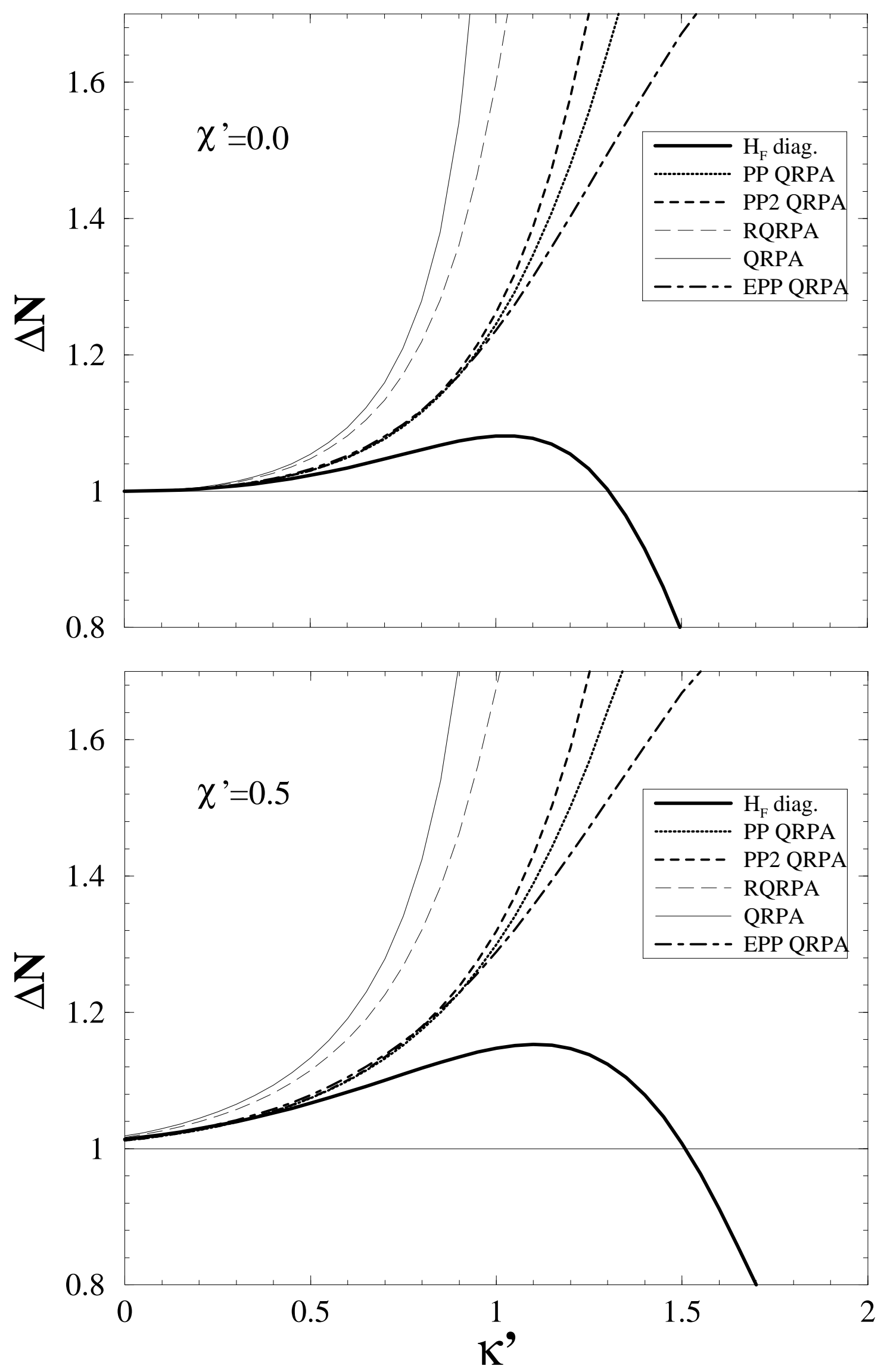

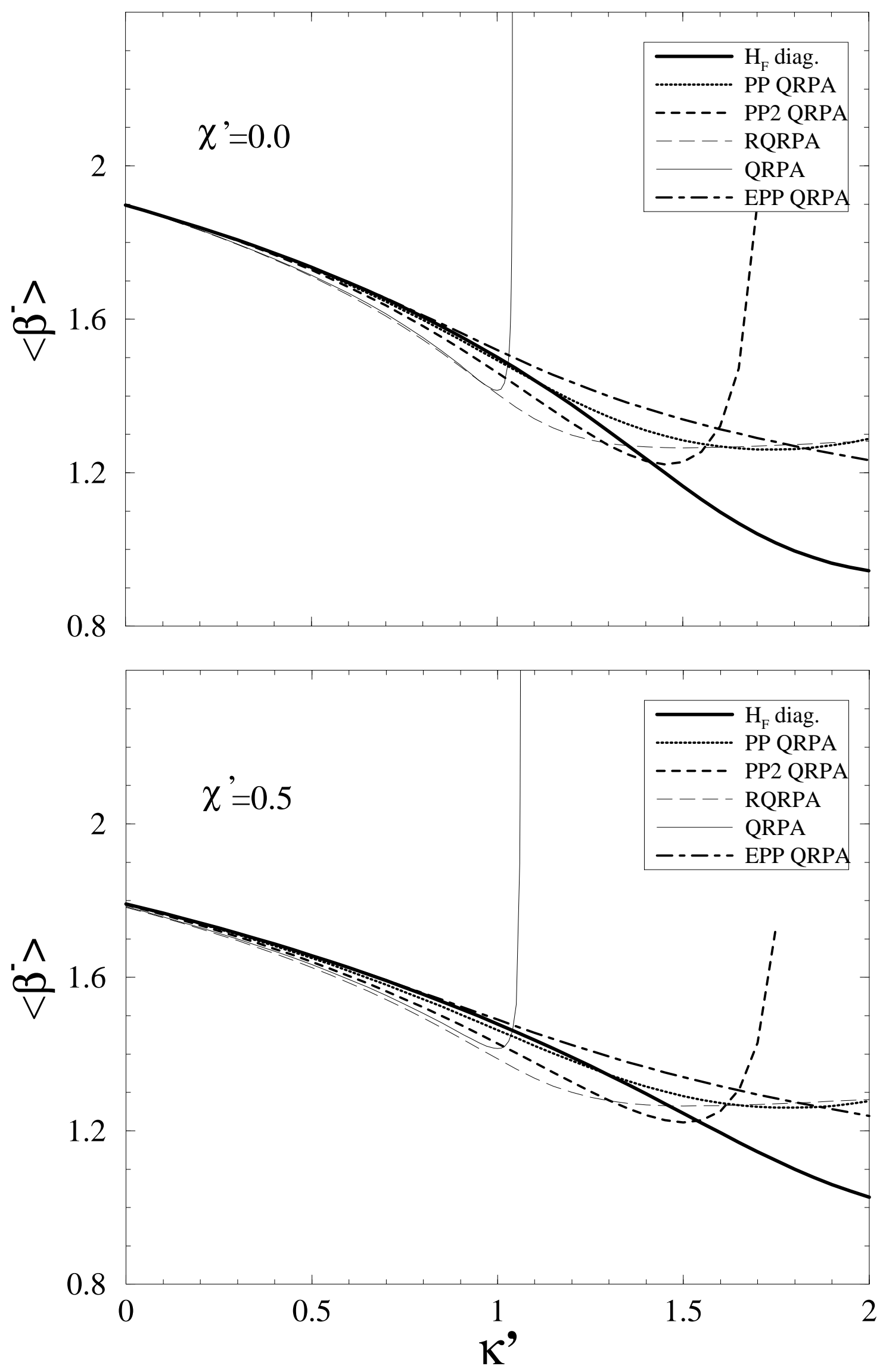

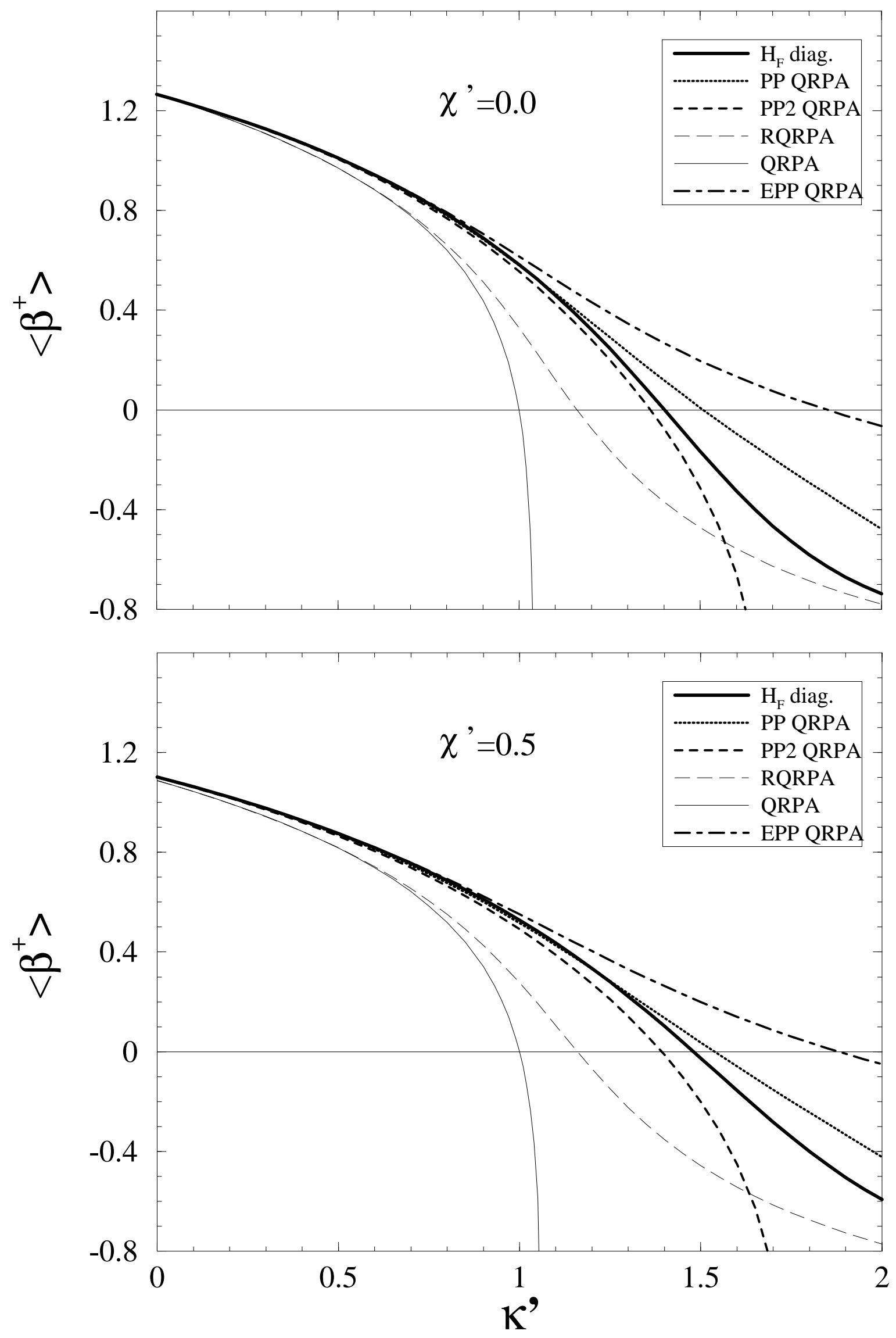

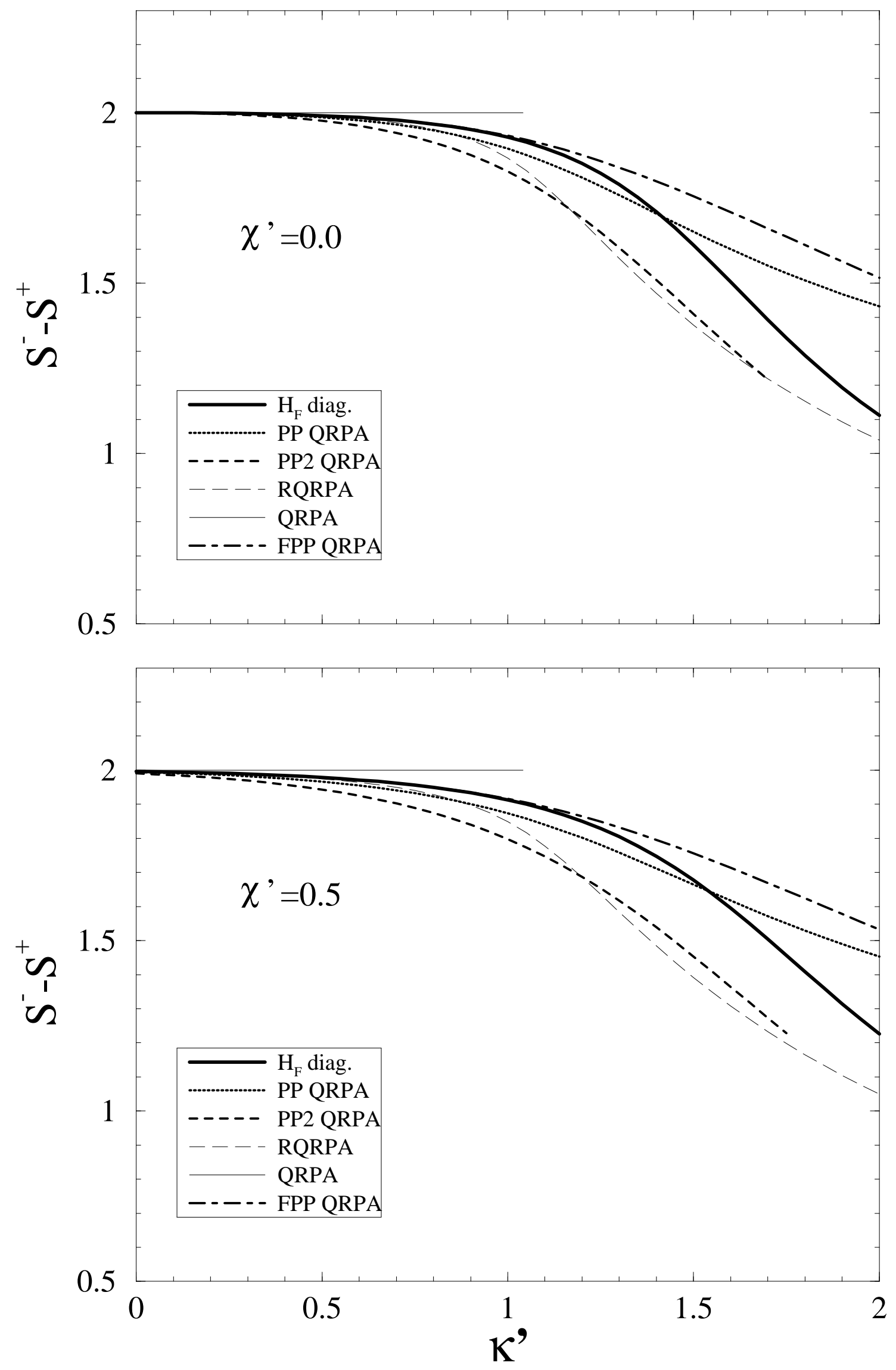

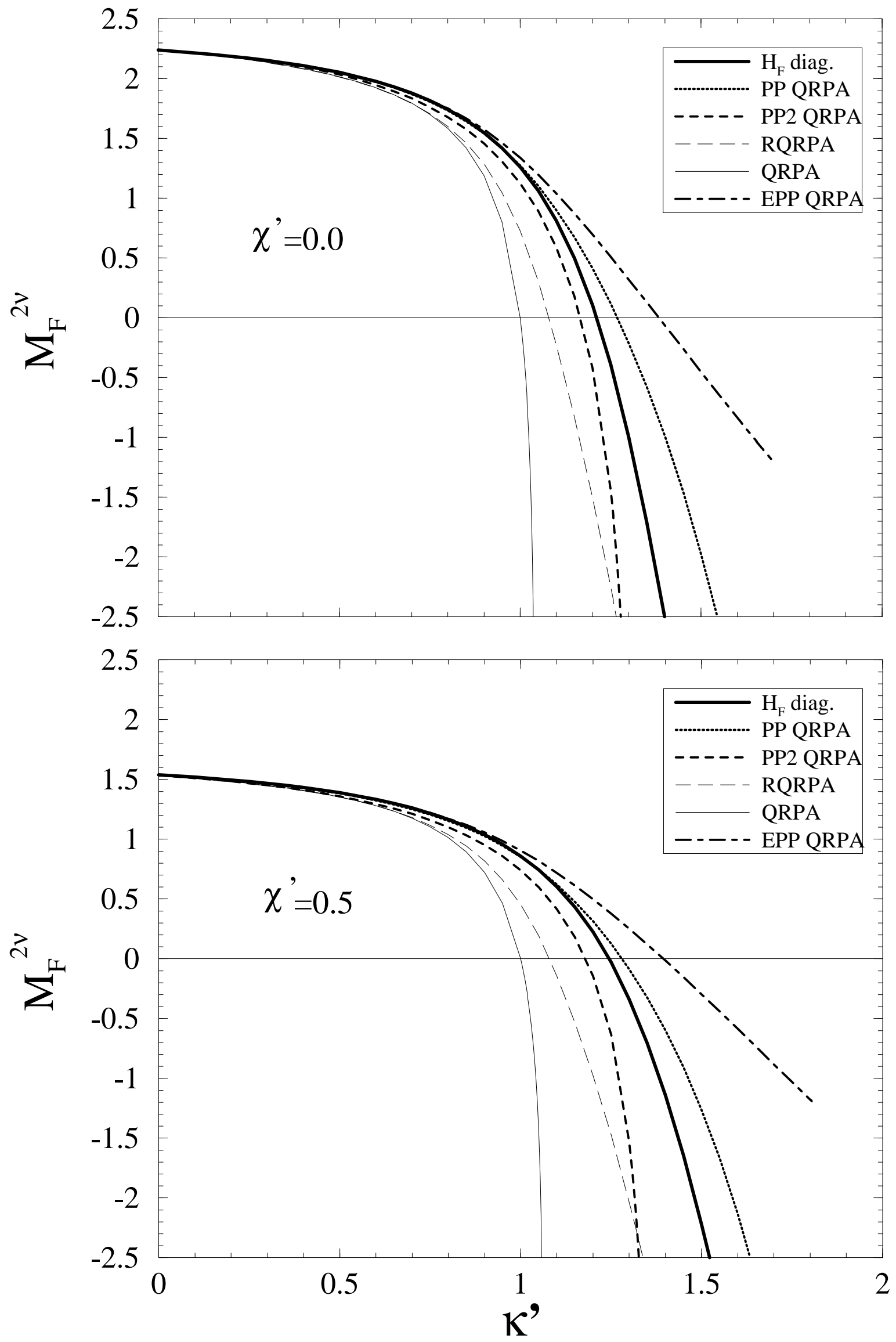